\title{
EFFECT OF DIFFERENT LIGHT CURING TECHNIQUES ON COLOR STABILITY OF TWO RESIN COMPOSITES (AN IN VITRO STUDY)
}

\author{
Fetouh El M*, Mohammed T M ${ }^{* *}$ and Hafez Th $\mathrm{A}^{* * *}$
}

\begin{abstract}
Purpose: The originality of the present study was to evaluate the effect of different light curing techniques on color stability of two resin composites. Methods the study samples were divided equally into two main groups according to the type of resin composite (microhybrid and nanohybrid) each main group was subdivided into four subgroups according to type of curing modes (halogen, LED in fast mode, LED in soft start mode and LED in pulse mode), Assessment of color was carried out using a portable reflective spectrophotometer over white background before curing, after finishing and polishing, after one and three months storage in artificial saliva at 37c Results after curing Both resin composite materials recorded statistically significant higher color change mean values above the clinically perceivable values varies between $\left(6.69^{\mathrm{B}} \pm 0.4\right.$ to $\left.12.58^{\mathrm{A}} \pm 0.51\right)$, after one month $\left(0.267^{\mathrm{B}} \pm 0.1\right.$ to $\left.3.847^{\mathrm{A}} \pm 0.14\right)$ and after three months $\left(0.46^{\mathrm{B}} \pm 0.07\right.$ to $\left.4.1^{\mathrm{A}} \pm 0.11\right)$ Conclusions Large color shift occur immediately after polymerization, this color change above the clinically accepted values ( $\Delta \mathrm{E}>3.3)$, Different LED curing modes affect the color stability of composite with nonsignificant difference and Color stability of composite depended mainly on material composition rather than curing modes.
\end{abstract}

\section{INTRODUCTION}

Resin composite has been considered the most popular esthetic restorative material due to its physico mechanical and esthetic properties. In view of this fact the clinician's attention has been directed towered the material color matching and color stability for getting a successful and longevity of the restoration ${ }^{(1)}$. Color stability of resin composites can be influenced by several extrinsic factors, such as intensity and duration of the polymerization reaction, exposure to environmental factors, including ambient radiation, heat changes, water and food colorants Moreover, also affected by intrinsic factors, such as the composition of the resin matrix, filler loading and particle size distribution, type of photoinitiator and percentage of remaining carbon bonds (2). It is known that the efficiency of polymerization may influence the discoloration. Since, the higher degree of monomer conversion, the lesser the residual monomers will be available to form colored degraded products ${ }^{(3)}$. The advantage of the light emitting diode (LED) curing unit are, high-power density which promotes a higher degree of monomer conversion and hence better results ${ }^{(4)}$, more spectral purity as it has a narrow band of light emission with wave length between 450 and $490 \mathrm{~nm}$, with peak emission at $470 \mathrm{~nm}$, which coincident with the absorption spectrum of most of the photoinitiators included in resin composite matrix ${ }^{(5)}$. while the halogen curing unite can promote the formation of polymer chains with lower molecular weight and residual monomers, consequently, partial polymerization reaction of the material will occur with part of the photoinitiator remaining idle ${ }^{(6)}$.

\section{MATERIALS AND METHODS}

\section{Resin Composite Materials}

Two different resin composite materials were selected for this study; micro-hybrid resin composite; Composan LCM (PROMEDICA, Domagkstr, Neumunster Germany) and Nano-hybrid resin composite Filtek ${ }^{\mathrm{TM}} \mathrm{Z} 250 \mathrm{XT}(3 \mathrm{M}$ ESPE, USA), as shown in Table $1 \&$ Figures (1 and 2).

\footnotetext{
* Demonstrator, Department of Operative dentistry, Faculty of Dental Medicine, Al-Azhar University (Assuit Branch)

** Professor of Operative dentistry, Faculty of Dental Medicine, Al-Azhar University Cairo Boys

*** Lecture of Operative dentistry, Faculty of Dental Medicine, Al-Azhar University (Assuit Branch)
} 


\section{Curing devices}

Two different types of curing devices were used in this study; Manufacture and information are listed in table (2) \& figures (3 and 4);

\section{Mold description}

A specially designed custom-made split circular Teflon mold with internal hole ( $2 \mathrm{~mm}$ height and $10 \mathrm{~mm}$ diameter) enclosed within teflon ring was used for specimen preparation in the form of discs

\section{Resin composite specimen's preparation}

The mold was placed on a transparent matrix strip supported by a glass slide and slightly overfilled with resin composite material. The mold and resin composite were covered by another matrix strip and glass slide. Light figure pressure was applied until the upper matrix strip and slide came into contact with the mold, the upper glass slid was removed and the color of resin composite was mea- sured by spectrophotometer directly through the matrix strip against white back ground

\section{2- Grouping of the specimens}

Resin composite specimens were divided into two main groups according to the type of resin composite used. Group RC1; prepared from composan LCM (Microhybrid) and Group RC2; prepared from Z250XT (Nanohybrid). Each main group was subdivided into four subgroups according to the type of curing techniques. Subgroup CT1 cured with halogen curing unit in high power mode (control), Subgroup CT2 cured with LED in fast or continuous mode, Subgroup CT3 cured with LED in ramp or soft start mode and Subgroup CT4 cured with LED in pulse mode, curing time was 40 seconds.

\section{3- Curing of resin specimens.}

Immediately following the color measurements, curing of the specimens were occurred through the matrix strip, the specimens were cured from the top

TABLE (1) Information's regarding the resin composite materials used for this study.

\begin{tabular}{|c|c|c|c|}
\hline Products & Classification & Composition & Manufacture \& patch No. \\
\hline Composan LCM & $\begin{array}{c}\text { Microhybrid } \\
\text { A3 }\end{array}$ & $\begin{array}{l}\text { Filler: } \\
76 \% \text { by Wt - } 60 \% \text { by Vol. } \\
\text { Composed of Microfillers approx } 0.05 \mu \mathrm{m} \\
\text { Small particle fillers approx } 0.5-2 \mu \mathrm{m} \\
\text { Monomers: } \\
\text { BIS-GAM, UDMA, TEGDMA, BHT and HEMA }\end{array}$ & $\begin{array}{l}\text { PROMEDICA, Domagk- } \\
\text { str, Neumunster, } \\
\text { German } \\
\text { info@ promedica.de } \\
\text { patch No. } \underline{2464}\end{array}$ \\
\hline Z250XT & $\begin{array}{c}\text { Nanohybrid } \\
\text { A3 }\end{array}$ & $\begin{array}{l}\text { Filler: } \\
81.8 \% \text { by Wt }-67.8 \% \text { by Vol. } \\
\text { Composed of Silica approx } 20 \mathrm{~nm} \\
\text { Zirconia/silica approx } 0.1-10 \mu \mathrm{m} \\
\text { Monomers: } \\
\text { BIS-GAM,UDMA } \\
\text { TEGDMA, PEGDMA and BIS-EMA }\end{array}$ & $\begin{array}{l}\text { 3M Espe,St Paul,USA } \\
\underline{\text { 3mespe.in@mmm. }} \\
\text { Com }\end{array}$ \\
\hline
\end{tabular}

TABLE (2) Types of curing devices used in this study

\begin{tabular}{|l|l|l|l|l|l|}
\hline Type of curing devices & Trade name & Tip diameter & Manufacture & Curing time & Light intensity \\
\hline Halogen & Blue Luxcer M-830 Monitex & $10 \mathrm{~mm}$ & Taiwan & $40 \mathrm{~s}$ & $600-800 \mathrm{~mW} / \mathrm{cm}^{2}$ \\
\hline LED (light emitting diode & D 2000 & $8 \mathrm{~mm}$ & Taiwan & $40 \mathrm{~s}$ & $1000-1200 \mathrm{~mW} / \mathrm{cm}^{2}$ \\
\hline
\end{tabular}




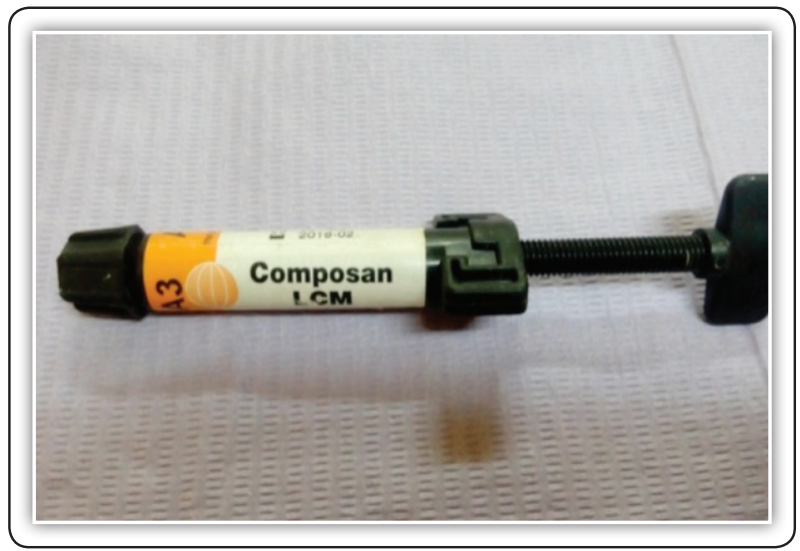

Fig. (1) Composan Micro-hybrid resin composite

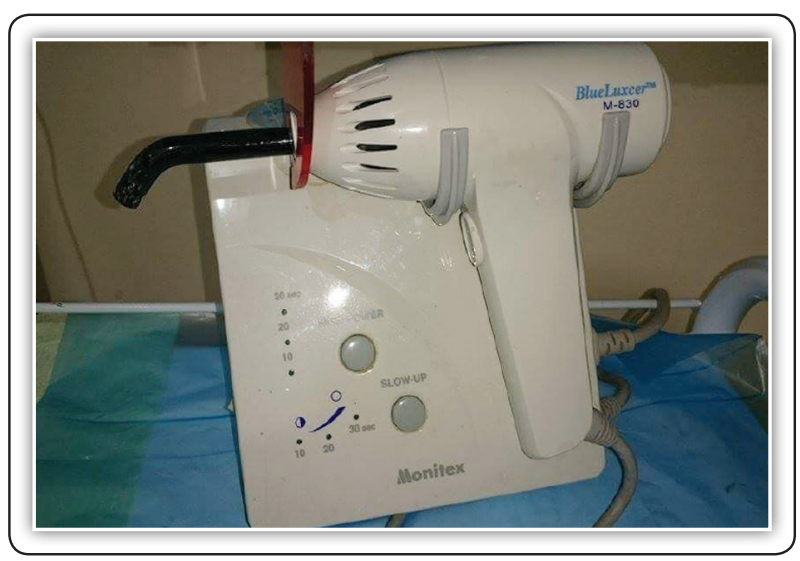

Fig. (3) Halogen light curing unit*

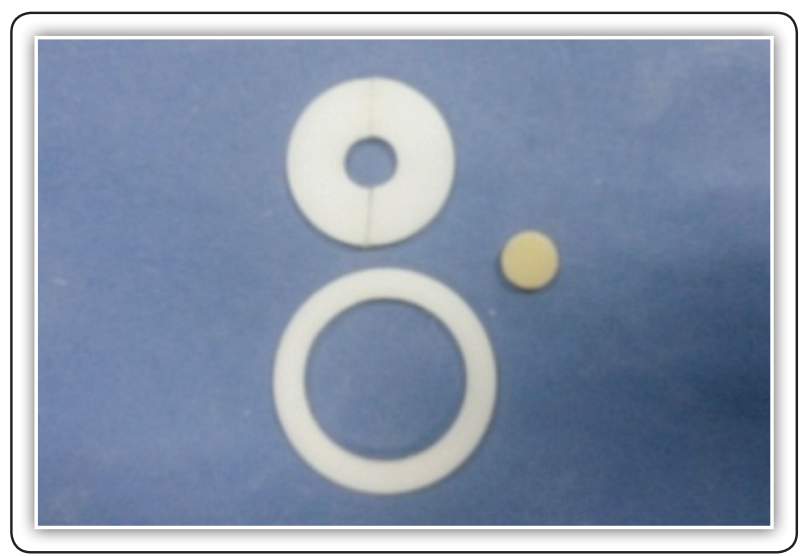

Fig. (5) custom-made split circular Teflon mold

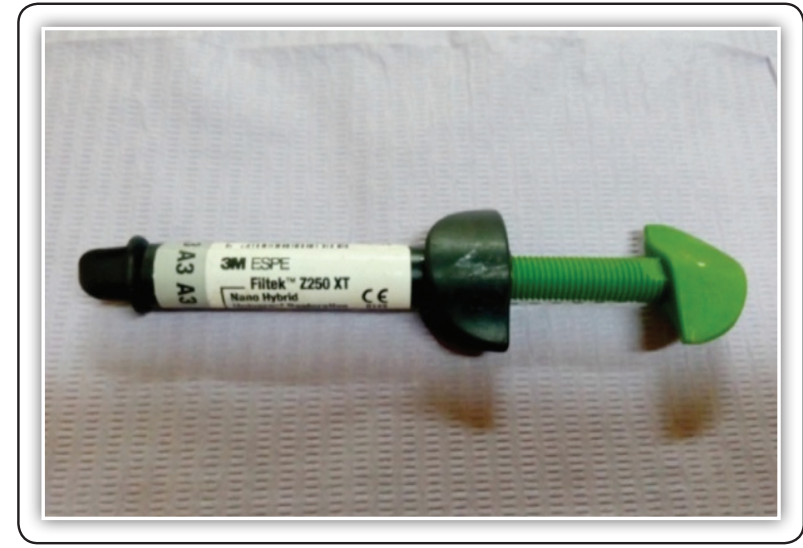

Fig. (2) Z250 nanohybrid composite

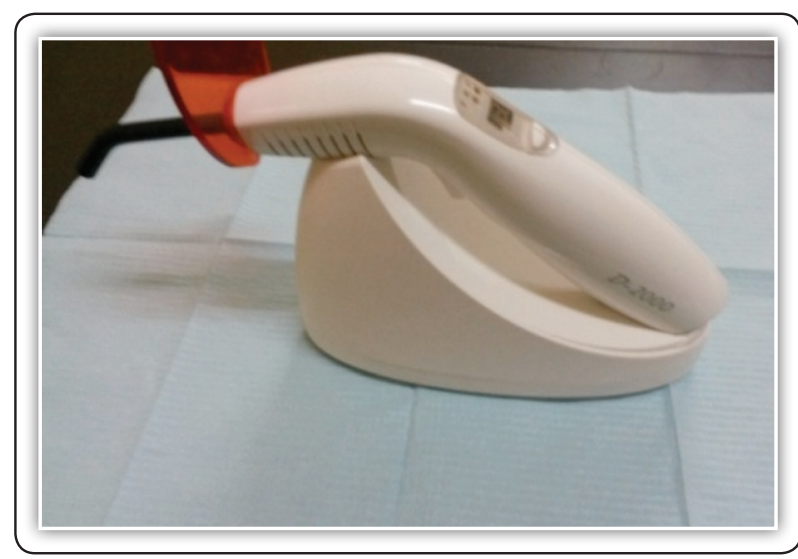

Fig. (4) LED light curing unit (D-2000)

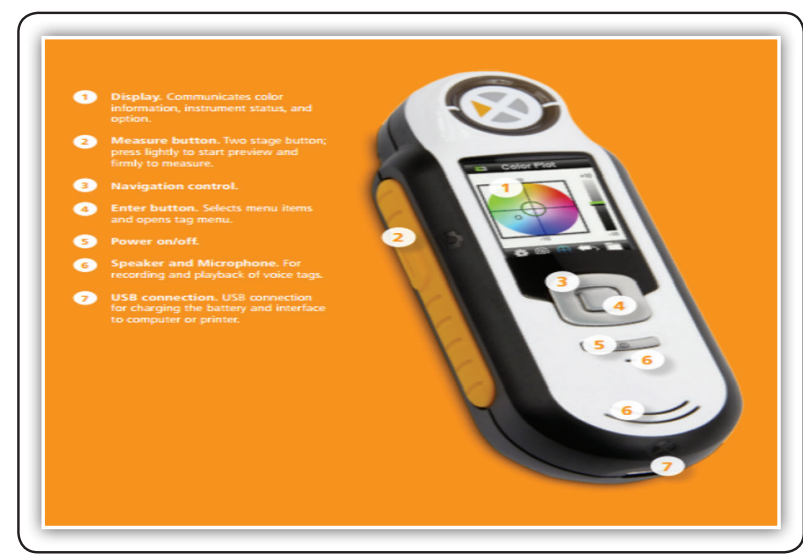

Fig. (6) Spectrophotometer 
surface only, the uncured surface of the specimens were marketed with No. 0.5 slow speed round bur. The mold was de assembled and the composite excess was removed with NO. 15 sharp scalpel

\section{4- Finishing of the specimens}

The cured surface of the specimens was finished and polished with soflex system according to manufacture instructions which beginning from coarse, medium, fine to super fine discs with low speed headpiece running at 5,000 rpm each disc was used for 15 seconds and changed after three samples. Resin composite discs were washed under running water to remove debris, then stored in de ionized distilled water and incubated for 24 hours at $37^{\circ} \mathrm{C}$

\section{5- Immersion of the specimens}

The specimens were immersed in artificial saliva and incubated at $37^{\circ}$ for three months, Color measurements were obtained after one and three months. Before each measurement the specimens were removed from artificial saliva, washed under running water, dried with gauze.

\section{6- Colorimetric measurements:}

The color of the specimens was measured using a portable Reflective spectrophotometer*. The aperture size was set to $4 \mathrm{~mm}$ and the specimens were exactly aligned with the device at the center. A white background was selected and measurements were made according to the CIE L*a*b* color space relative to the CIE standard illuminant D65. The color changes $(\Delta \mathrm{E})$ of the specimens were evaluated using the following formula: $\triangle \mathrm{E}(\mathrm{CIELAB})=$ $\left(\Delta \mathrm{L}^{* 2}+\Delta \mathrm{a}^{* 2}+\Delta \mathrm{b}^{* 2}\right)^{1 / 2}$

Where: $L^{*}=$ lightness $(0-100), a^{*}=($ change the color of the axis + red/ - green $)$ and $b^{*}=($ color variation axis + yellow/ - blue)

\section{Statistical analysis}

The collected data was calculated, tabulated and statistically analyzed. Data analysis was performed in several steps. Initially, descriptive statistics for each subgroup results. Two factors analysis of variance ANOVA test of significance was done for comparing variables affecting mean values (composite group and curing methods). One way ANOVA followed by pair-wise Tukey's post-hoc tests were performed to detect significance between curing subgroups. Student t-test was performed to detect significance between composite groups. Statistical analysis was performed using GraphPad Prism version 4.00 for Windows, GraphPad Software, San Diego California USA. P values $\leq 0.05$ are considered to be statistically significant in all tests.

\section{RESULTS}

\section{At no storage time}

Microhybrid resin composite cured with LED in pulse mode gave the highest mean values of color change ( $\mathrm{DE}=12.58 \pm 0.51)$ while the lowest mean values of color change $(\mathrm{DE}=6.69 \pm 0.4)$ was recorded for nanohybrid cured with halogen.

The difference between groups was statistically significant for both microhybrid and nanohybrid as indicated by ANOVA test $(\mathrm{p}<0.05)$.

Total effect of resin composites; Regardless to curing modes, it was found that microhybrid resin composite recorded statistically significant ( $\mathrm{p}<$ $0.05)$ higher color change mean value (11.6 DE) than nanohybrid (7.04 DE) as indicated two-way ANOVA test.

Total effect of curing modes; Irrespective of resin composite materials, totally it was found that LED in pulse mode recorded the highest color change means value (9.9) followed by LED in fast mode (9.8) then LED in soft start mode (9.7) while halogen recorded the lowest means value (7.7). The difference between curing modes was statistically significant as indicated by two way ANOVA test.

\section{One month storage time}

Halogen curing of microhybrid resin composite gave the highest mean value of color change 
TABLE (3): Mean and standard deviations of color stability at different storage times for resin composites cured with different curing techniques.

\begin{tabular}{|c|c|c|c|c|}
\hline Resin composites & Curing techniques & \multicolumn{3}{|c|}{ Storage times } \\
\hline \multirow{2}{*}{ RC } & CT & ST0 & ST1 & $4.1^{\mathrm{A}} \pm 0.11$ \\
\hline \multirow{3}{*}{ RC1 } & CT1 & $8.72^{\mathrm{B}} \pm 0.51$ & $3.847^{\mathrm{A}} \pm 0.14$ & $0.7^{\mathrm{B}} \pm 0.05$ \\
\cline { 2 - 5 } & CT2 & $12.56^{\mathrm{A}} \pm 0.29$ & $1.247^{\mathrm{BC}} \pm 0.01$ & $0.72^{\mathrm{B}} \pm 0.3$ \\
\cline { 2 - 5 } & CT3 & $12.38^{\mathrm{A}} \pm 0.24$ & $1.987^{\mathrm{B}} \pm 0.7$ & $0.65^{\mathrm{B}} \pm 0.38$ \\
\hline \multirow{3}{*}{ RC2 } & CT4 & $12.58^{\mathrm{A}} \pm 0.51$ & $1.083^{\mathrm{C}} \pm 0.6$ & $0.46^{\mathrm{B}} \pm 0.07$ \\
\cline { 2 - 5 } & CT1 & $6.69^{\mathrm{B}} \pm 0.4$ & $0.267^{\mathrm{B}} \pm 0.1$ & $0.6^{\mathrm{A}} \pm 0.2$ \\
\cline { 2 - 5 } & CT2 & $7.03^{\mathrm{AB}} \pm 0.26$ & $0.723^{\mathrm{A}} \pm 0.2$ & $0.52^{\mathrm{A}} \pm 0.23$ \\
\cline { 2 - 5 } & CT3 & $7.11^{\mathrm{AB}} \pm 0.02$ & $2.697^{\mathrm{A}} \pm 0.3$ & $0.64^{\mathrm{B}} \pm 0.24$ \\
\hline
\end{tabular}

Different letter in the same column indicating statistically significant difference

$(p<0.05) *$; significant $(p>0.05)$ ns; non-significant

(DE=3.847 \pm 0.14$)$ while the lowest mean value of color change $(\mathrm{DE}=0.267 \pm 0.1)$ was recorded for nanohybrid cured with halogen

The difference between groups was statistically significant for both microhybrid and nanohybrid as indicated by ANOVA test $(\mathrm{p}<0.05)$.

Total effect of resin composite Regardless to curing modes, it was found that microhybrid resin composite recorded statistically significant $(\mathrm{p}<$ $0.05)$ higher color change mean value (2.04) than nanohybrid resin composite (1.1 DE) as indicated two-way ANOVA test.

Total effect of curing modes; Irrespective of resin composite materials, totally it was found that LED in soft start mode recorded the highest change means value (2.3) followed by halogen (2.1) then LED in fast mode (0.985) while LED in pulse mode recorded the lowest means value (0.81). The difference between curing modes was statistically significant as indicated by two way ANOVA test.

\section{Three months storage times}

At three months storage in artificial saliva; halogen curing of microhybrid resin composite recorded the highest mean value of color change $(\mathrm{DE}=$ $4.1 \pm 0.11)$ while halogen curing of nanoohybrid resin composite recorded the lowest $(\mathrm{DE}=0.46 \pm 0.07$ ).

The difference between groups was statistically significant for both microhybrid and nanohybrid as indicated by ANOVA test $(\mathrm{p}<0.05)$.

Total effect of resin composites; Regardless to curing modes, it was found that microhybrid resin composite recorded statistically significant $(\mathrm{p}<$ $0.05)$ higher change mean value (1.5) than nanohybrid (1.1) as indicated by two way ANOVA test.

Total effect of curing modes; Irrespective of resin composite materials, totally it was found that halogen recorded the highest change means value (2.2) followed by LED in soft start mode (1.7) then LED in fast mode (0.7) while LED in pulse mode recorded the lowest means value (0.6). The difference between curing modes was statistically significant as indicated by two way ANOVA test. 


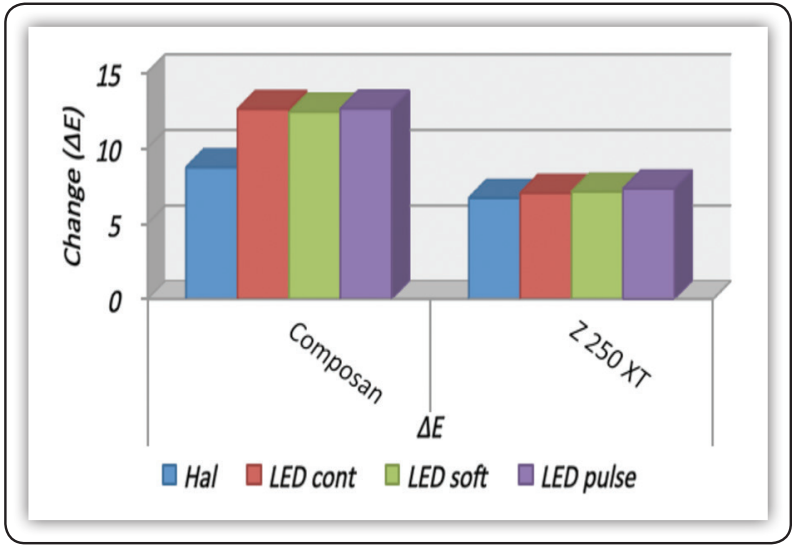

Fig. (7) Histogram of the mean values of color change (DE) for both groups as function of curing

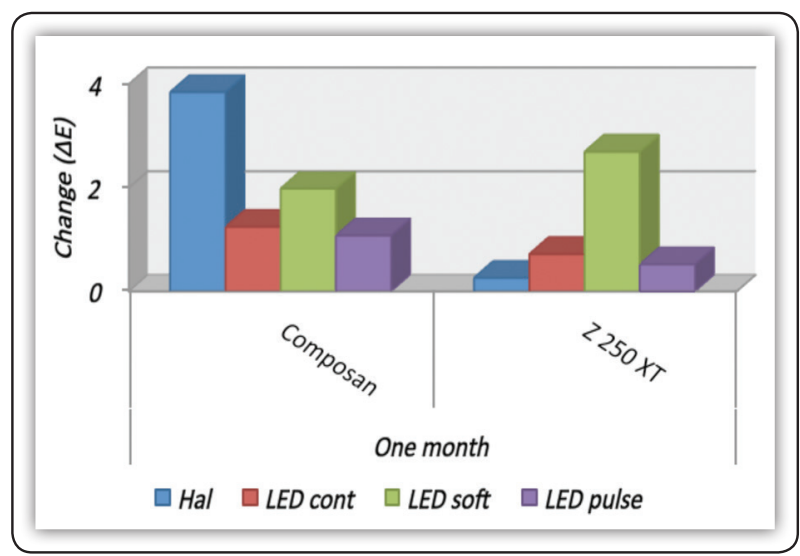

Histogram of the mean values of color change (DE) for both groups after one-month storage in artificial saliva

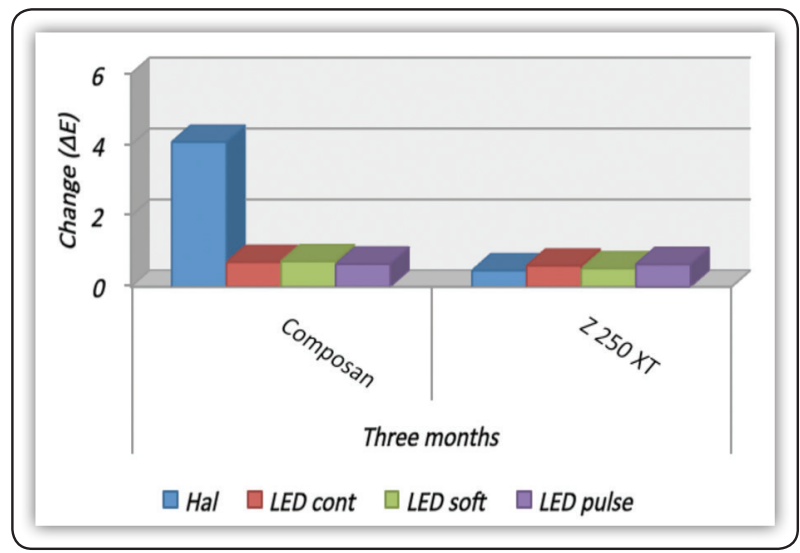

Fig. (9) Histogram of the mean values of color change (DE) for both groups as function of curing after three months storage in artificial saliva

\section{DISCUSSION}

Increasing aesthetic demands from patients has resulted in an increasingly widespread use of resin composites in dental practice. The physical, mechanical, aesthetic properties and clinical behavior of resin composites depend on their structures $^{(7)}$. Color plays an important role in obtaining optimum esthetics the major disadvantage of resin composites is their color instability which may be the major cause for replacement of restorations, color stability of a composite is related to the resin matrix, dimensions of filler particles, depth of polymerization and coloring agents ${ }^{(8)}$. Adequate polymerization and degree of monomer conversion are a crucial factor for obtaining optimal physical and clinical performance of any hybrid resin composite ${ }^{(9)}$. Inadequate polymerization results in inferior physio-mechanical properties such as color instability and poor wear resistance, adverse tissue reaction, increased water sorption and solubility resulting in an early failure of the restoration (10). Discoloration can be evaluated by visual or instrumental techniques; the color evaluation by visual comparison may not be a reliable method due to inconsistencies inherent in color perception and specification amongst observers. Instrumental techniques for color measurement include colorimetry, spectrophotometry and digital image analysis of which spectrophotometry has been reported to be a reliable technique in dental material studies. ${ }^{(11)}$. All resin composite specimens recorded statistically significant higher color change mean values above the clinically perceivable value $\Delta \mathrm{E}$ $>3.3$ after polymerization, This in agreement with Emine $\mathrm{S}$ et $\mathrm{al}^{(12)}$, CAMILA $\mathrm{S}$ et al ${ }^{(13)}$ who reported that higher color change mean values occurred immediately after polymerization they explain the reason for increase in $\Delta \mathrm{E}$ might be attributed to the shift in optical properties of the resin known to take place during the cross-linking of the monomers into polymeric chains as the polymerized resin tend to have greater diffuse reflectance than unpolymerized 
resin this alteration reflects the increase in reflective index of the resin phase associated with monomer conversion while the reflective index of the filler remain unchanged. After one and three months storage in artificial saliva all resin composite specimens recorded statistically non significant color changes below the clinically perceivable values except for group microhybrid resin composite cured with halogen which recorded the highest $\Delta \mathrm{E}\left(3.847^{\mathrm{A}} \pm 0.14\right)$ after one month and after three months $\left(4.1^{\mathrm{A}} \pm 0.11\right)$, This result in accordance with the study of Dalia, M et $\mathrm{al}^{(14)}$ who found that, no statistically significant difference between LED and QTH on permuting color change of nano filled and microhybrid composites, nano filled demonstrated less color change than micro hybrid, they attributed their finding to resin composition, and in agreement with Patricia A et $\mathrm{al}^{(15)}$, they reported that nanofilled composite recorded color change below the clinically accepted values when stored in artificial saliva for two months, Joseph A et al ${ }^{(16)}$ reported that nanohybrid composite cured with QTH and stored in distilled water was more color stable than microhybrid and submicron, also in agreement with Iffat $\mathrm{N}$, et $\mathrm{al}^{(17)}$, they reported that nanohybrid resin composite cured with QTH or LED and stored in distilled water for one month was more color stable than hybrid composite, no significant difference between QTH and LED on permuting color change, the authors referred their finding to staining susceptibility of resin composites after storage in artificial saliva is related to water sorption as the artificial saliva contain no pigments, this water sorption is directly related to composite composition and surface properties, the composition and size of the filler particles affects the surface smoothness and susceptibility to extrinsic staining, therefore, it can be expected that composite with smaller particles size will have smoother surface and will retain less surface stains than composite with large particles size and rough surfaces ${ }^{(18)}$.

\section{CONCLUSIONS}

1- Large color shift occur immediately after polymerization for both composite materials, this color change above the clinically accepted values $(\Delta \mathrm{E}>3.3)$.

2- Artificial saliva stain the composite below the clinically accepted values

3- Different LED light curing modes affect the color stability of composite with non-significant difference.

4- Color stability of composite depended mainly on material composition rather than curing modes.

\section{REFERENCES}

1- Puckett A, Fitchie J, Kirk P, Gamblin J. Direct composite restoration materials. Dent Clinic North Am. 2007; 51: 659-75.

2- Fontes S, Fernández M, Moura C, Meireles S. Color stability of a nanofill composite: effect of different immersion media. J Appl Oral Sci. 2009; 17:388- 91.

3- Domingos P, Garcia P, Oliveira A, Palma D. Composite resin color stability influence of light source and immersion media. J Appl Oral Sci. 2011; 19:204-11.

4- Samra A, Pereira S, Delgado L, Borges C. Color stability evaluation of esthetic restorative materials. Braz Oral Res.2008; 22:205-10.

5- Wiggins M, Hartung M, Althoff O, Wastian C, Mitra S. Curing performance of a new-generation light-emitting diode dental curing unit. J Am Dent Assoc. 2004; 135:1471-9.

6- Patel S, Gordan V, Barret A, Shen C. The effect of surface finishing and storage solutions on the color stability of resin based composite. J Am Dent Assoc. 2004; 135:587-94.

7- Inokoshi S, Burrow MF, Kataumi M, Yamada T \& Takatsu T. Opacity and color changes of tooth-colored restorative materials Oper Dent. 1996; 21(2): 73-80.

8- Shekhar B, Shivani K, Ratnasothy S, Color Changes of tooth colored restorative dental materials immersed in food simulating solution. J Dent Mater. 2012; 1-6. 
9- Behnaz E, Shaghayegh R, Mahdie P, Ali B, Hoda A, Comparative evaluation of the effect of light emitting diode (LED) and quartz tungsten halogen (QTH) light curing units on color stability of Filtek Z350 XT. Caspian J Dent Res.2014; 3 (1):41-6.

10- Alexandre L, Anna K, Guilherme S, Paula C, Alessandra B, Sigmar M. Color stability of composites effect of immersion media. Acta Odontal Latinoam.2011; 24:(2):193-9.

11- Diab M, Mubarak E and Olaa M, Effect of five commercial mouth rinses on the microhardness and color stability of two resin composite restorative materials. Aust J Basic Appl Sci.2007; 1(4): 667-74.

12- Emine S, Mehmet B, Ertan E, Mehmet A, Aslihan U, Assessment of changes in color and color parameters of light-cured composite resin after alternative polymerization methods Eur J Dent. 2013;(7):110- 6.

13- Camila S, Marc C, Jamil A, Color stability of ten resinbased restorative materials. J EsthetRestor Dent. 2012;
Vol. 2: No 3: 185-99.

14- Dalia M, Ola I, Hebat-Allah M, Ahmed A, In Situ Investigation on color change of resin composite restoratives cured by two different curing units. J Am Sci. 2012; 708-15.

15- Patricia A, Patrícia P, Ana Luisa B, Regina G, Composite resin color stability: influence of light sources and immersion media. J Appl Oral Sci. 2011; 19(3):204-11.

16- Joseph A, Huan Lu, J. Todd Milledge, Lilin H, John P, In vitro staining of resin composites by liquids ingested by children. Pediatr Dent.2008; 30: (4): 317-22.

17- Iffat Nasim, Prassana Neelakantan, R.Sujeer, C.V. Subbarao, Color stability of micro filled, micro hybrid and nano composite resin an in vitro study. J Dent.2010; 137-42.

18- Fabiana M, João T, Larissa P, Terezinha de, Regina G, Linda W, Evaluation of translucency of a nanofilled and a microhybrid resin composites. Braz Dent Sci. 2012; 15 (3): $38-42$ 